\title{
Longitudinal Assessment of Systemic Steroid Therapy on Hyperinflammatory Endothelial Biomarker Profiles and Serology Responses of COVID-19 Patients
}

Jonathan Thomas Sims

Eli Lilly and Company https://orcid.org/0000-0001-8599-2157

Ching-Yun Chang

Eli Lilly and Company

Josh Poorbaugh

Eli Lilly and Company

Montanea Daniels

Eli Lilly and Company

Stephanie Beasley

Eli Lilly and Company

Lin Zhang

Eli Lilly and Company

George Rodgers

Eli Lilly and Company

\section{Fabio Lena}

Misericordia Community Hospital

Leonardo Lacerenza

Misericordia Community Hospital

\section{Bruno Sposato}

Misericordia Community Hospital

Annabelle Dupont

Institut Pasteur de Lille

\section{Sophie Susen}

Institut Pasteur de Lille

\section{Giacomo Casalini}

University of Milan Department of Clinical Sciences Luigi Sacco: Universita degli Studi di Milano Dipartimento di Scienze Biomediche e Cliniche Luigi Sacco

\section{Mario Corbellino}

ASST Fatebenefratelli Sacco: Aziende Socio Sanitarie Territoriale Fatebenefratelli Sacco 
Imperial College London

Venkatesh Krishnan ( $\sim$ krishnan_gary@lilly.com )

Eli Lilly and Company

\section{Research Article}

Keywords: COVID-19, CXCL9, Cytokine, Dexamethasone, Inflammation, Serology

Posted Date: January 27th, 2022

DOI: https://doi.org/10.21203/rs.3.rs-1217324/v1

License: (c) (1) This work is licensed under a Creative Commons Attribution 4.0 International License. Read Full License 


\section{Abstract}

This first of its kind study provides objective context to the potential mechanism of action of corticosteroid use in COVID-19 patients from 3 separate European medical centers by connecting inflammatory biomarkers to IgG levels for the SARS-CoV-2 spike protein antigens and neutralization of ACE2 binding within infected individuals. CXCL9 is described herein as an important COVID-19 biomarker connecting disease severity with inflammatory biomarker and serology response profiles in corticosteroidtreated patients.

\section{Full Text}

This first of its kind study provides objective context to the potential mechanism of action of corticosteroid use by connecting inflammatory biomarkers to IgG levels for the SARS-CoV-2 spike protein antigens and neutralization of ACE2 binding within patients across 3 institutions from Italy and France who received corticosteroids (dexamethasone, $n=5$, or prednisolone, $n=1$ ) or usual standard of care (SOC, $\mathrm{n}=22$ ) therapy. The median Ordinal Scale (WHO) upon admission was OS-5 and no difference in days from symptom onset or Ordinal scale at study entry was observed between these 2 groups (Supplementary Table 1, Supplementary Figure 1). Utilizing Olink multiplex technology and IL-19 ELISA, we assessed 185 analytes in the circulation of COVID-19 patients along with Luminex-based measurement of 10 immunoglobulins, including neutralization assessment.

We observed prominent dysregulation of IL-8, CCL7/MCP-3, S100A12/ENRAGE, and IL-6 in the circulation of these patient cohorts relative to 12 age/sex-matched healthy controls (HC) (Figure 1A). IL-6 correlated modestly with baseline Ordinal scale and the broader inflammatory biomarker profile was characterized by strong correlations to circulating neutrophils and serum creatinine (Figure 1A). Levels of TNFRSF10A, IL-10RA, CXCL9, TRAILR2, IL-18, and TNFa negatively correlated with SpO2 percentage and positively correlated with Ordinal Scale at admission emphasizing the potentially important role of these molecular pathways within hospitalized patients (Figure 1A) [1].

Focusing on markers differentially regulated in COVID-19 patients, we observed a unique biomarker profile in patients depending on their therapeutic journey. Analytes elevated in baseline COVID-19, FGF21, CCL3, CCL4, CEACAM8, CCL13/MCP4, IL-19, IL15RA, ANGPT1, MMP7, and CXCL9, all decreased more in corticosteroid-treated patients relative to SOC (Figure 1A).

We observed serology titers for all SARS-CoV-2 antigens with RBD mutant proteins increasing or remaining relatively unchanged within steroid-treated patients relative to the SOC-treated patients over the course of 7 days in this small study, mirrored by an increase in neutralization of the RBD-ACE2 interaction in the same steroid-treated patients (Figure 1B). Further research into the mechanism of action of corticosteroid-reduced IgG levels will need to occur, but in this study, there was no detrimental impact on antibody responses to COVID-19 infection. Within these patients, repeated measured correlations demonstrated inverse correlation of ACE2 neutralization IC50 not only with ACE2, but also 
TNFSF11/TRANCE protein in the serum (Figure 1B). This data argues that circulating antibodies with more potent neutralization (lower IC50) are found in patients with higher serum levels of ACE2 and TNFSF11/TRANCE, the former known to be shed by interactions with viral spike protein and the latter to decrease when viral pathogenesis is most robust [2,3]. Related to the broad suppressive nature of corticosteroids, certain analytes reduced in circulation of COVID-19 patients, such as SCF and GDF2, were inversely correlated with serological IgG response markers (Figure 1B). Intriguingly, a unique inflammatory biomarker profile was observed to correlate with the seasonal coronavirus protein control (NL63) relative to the profile linked to SARS-CoV-2 antigens and related RBD mutant proteins (Figure 1B). Positive correlations were observed for IL-27, MERTK, IL-17A, CXCL9, CCL3, GAL9, TNF, CCL4, FGF21, VSIG2, DCN, CX3CL1, IL-19, CASP8, IL-10, AGRP, FABP2, CXCL10, CD84, SCF, and IFNg with ACE2 neutralization IC50 values (Figure 1B). Higher ACE2 neutralization IC50 values indicate less potency of the antibody response and hence the observed increase in many proinflammatory signals (e.g. IL-19, TNF, CXCL9, and IFNg). This tight regulatory phenomenon between inflammatory biomarkers and serology was clearly evident within the tested timeframe of 7 days post corticosteroid treatment.

The correlation of COVID-19 inflammatory markers, which we previously linked to severe disease (e.g., IFNg, TNF, CXCL9, and IL-10), with higher IC50 neutralization values may demonstrate poor levels of ACE2/RBD interference in patients experiencing the hyperinflammatory endothelial-linked cytokine storm of COVID-19 [4].

Importantly, the elevation of markers indicative of innate immune activation (CCL13/MCP4, CCL3, CCL4, CXCL9) are reduced in steroid-treated patients. We observed that CXCL9 is an important COVID-19 biomarker describing immunological responses in corticosteroid-treated patients based on its correlation to Ordinal scale at baseline, decreased levels over time in corticosteroid-treated patients, and correlation with ACE2 neutralization IC50 values; therefore, warranting further evaluation and clinical monitoring within patients treated with corticosteroids. However, key systemic inflammatory cytokines linked to adaptive immune responses elevated in COVID-19 vs HC are not differentially regulated in steroid vs SOCtreated patients, demonstrating that early effective targeted therapies against these will be most successful in rapidly reducing the inflammatory burden that severe patients experience.

\section{Methods}

\section{Patients and Samples}

This study included a cohort of 28 patients requiring supplemental oxygen. 6 received corticosteroids (Steroids) prior to first sample collection, while 22 received a variety of standard of care (SOC) therapeutic regimen ranging from antibiotics to antivirals (Supplementary Figure 1A). The median Ordinal Scale (WHO) upon admission was OS5. No difference was observed in Ordinal scale at admission or mean time to symptom onset between Steroids or SOC groups (Supplementary Figure 1B, C). 12 age/sex-matched healthy controls were included for comparison.

\section{ELISA and multiplex immunoassays}


Protein Biomarker Assessments

Serum samples were analyzed with the Olink Inflammation I and Cardiovascular II Proseek multiplex assays (Uppsala, Sweden), a proximity extension assay (PEA) technology that utilizes oligonucleotidelabeled antibody probes, according to manufacturer's specifications. The levels of 184 analyte-specific deoxyribonucleic acid (DNA) amplicons were quantified for each patient on the Fluidigm Biomark HD (San Francisco, CA). IL-19 was assessed as previously described [4].

\section{Luminex Serology and Neutralization methodology}

Fluorescently-dyed MagPlex-Microspheres (Luminex XMAP) were used to separate multiple conjugated protein antigens into spectrally distinct regions allowing for the simultaneous detection and quantitation of antibodies against these proteins. Additionally, endogenous neutralization of the spike protein in patient sample was measured by detecting the ability of a PE-labeled RBD to bind to a recombinant ACE2-conjugated bead.

For serological/neutralization testing, serum samples were titered in phosphate buffered saline-high salt solution (PBS-HS; $0.01 \mathrm{M} \mathrm{PBS,} 1 \% \mathrm{BSA}, 0.02 \%$ Tween, $300 \mathrm{mM} \mathrm{NaCl}$ ) and mixed with antigen-conjugated microspheres (4 SARS-CoV-2 antigens (NTD, RBD1, RBD2, and full length spike (ST4)), 5 mutant proteins (RBD-F490S, RBD-N460K, RBD-E484Q, RBD-Q493R, and full length spike D614G) and a seasonal coronavirus family member (NL63), as well as phycoerythrin (PE)-conjugated RBD antigen (50ng/mL)) for 45 minutes (Supplementary Table 2). Subsequently, beads were separated from supernatant using a magnet with 75uL transferred to a new plate containing ACE2-conjugated beads. The remaining beads were washed and combined with secondary PE-conjugated antibody (algG, \#109-115-098, Jackson Labs). Both plates were incubated for 45 minutes at RT. Finally, plates were washed ( $3 x)$ with PBS-low salt solution (PBS-LS; 0.01 M PBS, 1\% BSA, 0.02\% Tween) and read using a Luminex FlexMap3D System.

\section{Statistical Analyses}

Titer is commonly defined as the smallest dilution above the cut point or the dilution factor at the cut point based on an interpolation of assay values that straddle the cut point. In the serology assay, we used the latter method to calculate the titers. To calculate potency of the neutralization component of the Luminex assay, a 4-parameter logistic regression was used to calculate the absolute IC50. Samples with negative maximum percentage inhibition were excluded from further analysis, while IC50s of samples with maximum percentage inhibition less than $50 \%$ were imputed to a large number.

Spearman correlation coefficient was calculated between the titers, IC50s, protein biomarkers, and clinical measures accounting for multiplicity adjustment and false discovery rate as previously described [4]. A mixed-effect model was used to assess time effect of treatment accounting for age, sex, and multiplicity adjustments as previously described [4].

\section{Declarations}


Ethics approval and consent to participate: All consent forms and protocols were approved by appropriate ethical review boards prior to study initiation.

Consent for publication: Not applicable.

Availability of data and materials: The data from the current study are available from the corresponding author on reasonable request.

Competing interests: Jonathan T. Sims, Venkatesh Krishnan, Ching-Yun Chang, Josh Poorbaugh, Montanea Daniels, Stephanie L. Beasley, Lin Zhang, \& George H. Rodgers are employees and may be shareholders of Eli Lilly and Company. Fabio Lena, Leonardo G. Lacerenza, Bruno Sposato, Annabelle Dupont, Sophie Susen, Giacomo Casalini, and Mario Corbellino report no conflicts of interest. Justin Stebbing is editor-in-chief of Oncogene, sits on several scientific advisory boards, including Benevolent Al, and consults with Lansdowne partners, Vitruvian, and Eli Lilly and Company, and sits on the Board of Directors for BB Biotech Healthcare Trust.

Funding: The analytical measures using Olink within the study were funded by Eli Lilly and Company. The physicians and the COVID-19 patients did not receive any funding or reimbursement from Eli Lilly and Company.

Authors' contributions: J.T.S., V.K., J.S., M.C., F.L., and S.S. conceived the study. J.T.S., J.P., M.D., S.L.B., and G.H.R. conducted the laboratory experiments. J.T.S., C-Y.C., V.K., J.P. and L.Z. designed and conducted the main analyses and interpreted the results. J.P., G.H.R., F.L., L.G.L, B.S., A.D., S.S., G.C., M.C. and J.S. also contributed to the discussion of analyses. J.T.S. and V.K. wrote the first draft of the manuscript. J.T.S., J.P., J.S., and V.K. wrote the manuscript, with contributions and review by all other authors.

Acknowledgments: The authors would like to thank the continued dedication of healthcare workers across the world for their response to COVID-19. We are indebted to Julien Poissy, Carlotta Pucci, Michele Bindi, Cesira Nencioni, Leonardo Croci, and Maja Rossi for collaboration and assistance with this study. We thank Jeffrey Boyles and Denisa Foster for generating recombinant proteins for the serology assay. We thank Autum Auxier and Tomasz Izbicki for sample management and Robert Benschop for scientific input and support.

\section{References}

1. Ye Q, Wang B, Mao J. The pathogenesis and treatment of the 'Cytokine Storm' in COVID-19. J Infect. 2020;80(6):607-13.

2. Jia H, Neptune E, Cui H. Targeting ACE2 for COVID-19 Therapy: Opportunities and Challenges. Am J Respir Cell Mol Biol. 2021;64(4):416-25.

3. Haljasmägi $L$, et al. Longitudinal proteomic profiling reveals increased early inflammation and sustained apoptosis proteins in severe COVID-19. Sci Rep. 2020;10(1):20533. 
4. Sims JT, et al. Characterization of the cytokine storm reflects hyperinflammatory endothelial dysfunction in COVID-19. Journal of Allergy Clinical Immunology. 2021;147(1):107-11.

\section{Figures}

Figure $1 \quad F C$ at least 1.5 fold and Corr $>0.3$ and significant

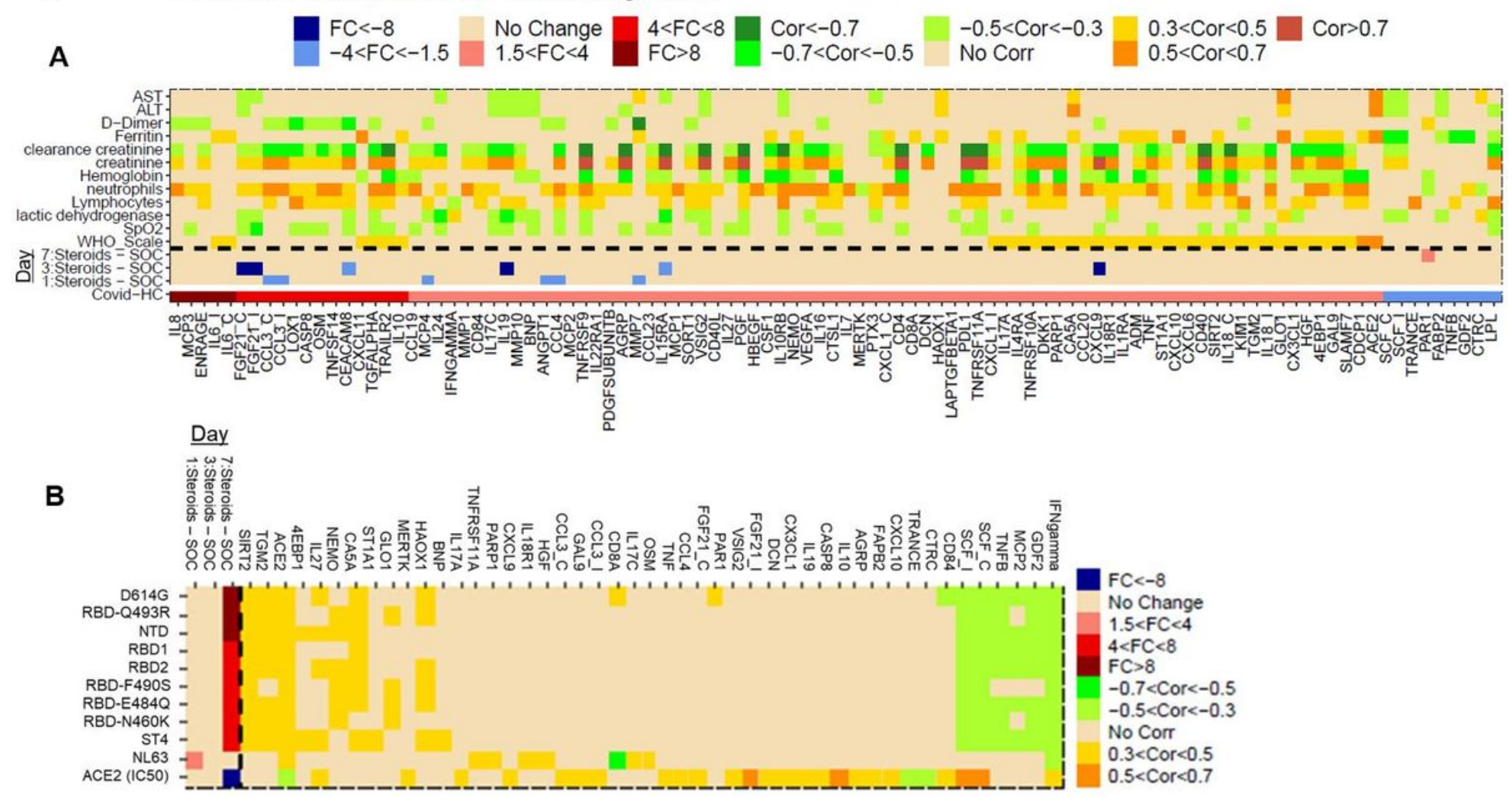

\section{Figure 1}

A-lower) Heatmap analysis comparing fold change for protein biomarker levels in baseline samples of COVID-19 patients versus age/sex-matched healthy controls (HC). Analytes are ordered along the $x$-axis by $\mathrm{P}$-value significance $(\mathrm{P}<.05)$ based on their positive and negative $\mathrm{FC}(>1.5 \mathrm{x})$ relative to $\mathrm{HC}$. A-middle) Fold change of analytes dysregulated in baseline COVID-19 patients relative to HCs over time between Steroid and SOC treated patients. A-top) Correlations of biomarkers and clinical assessments (i.e., WHO Ordinal scale, inflammatory signals (D-dimer, Ferritin), and blood cell counts) are shown for baseline samples of COVID-19 patients. B-left) Heatmap analysis of fold change of IgG levels for the SARS-CoV-2 spike protein antigens over time between Steroid and SOC treated patients. B-right) Correlations of biomarkers with IgG levels for SARS-CoV-2 spike protein antigens over time. Red or blue represents significantly upregulated or downregulated markers, respectively, between 1.5x-4x (light shade), 4x-8x (medium shade), or $>8 x$ (dark shade). Orange or green represents significantly positive or negative correlated markers, respectively, between $0.3<$ Cor $<0.5$ (light shade), $0.5<$ Cor $<0.7$ (medium shade), or Cor $>0.7$ (dark shade). "_I" or "_C" refers to analytes measured by Olink Inflammation I panel or Cardiovascular II panel, respectively. 


\section{Supplementary Files}

This is a list of supplementary files associated with this preprint. Click to download.

- SuppTable1.jpg

- SuppTable2.jpg

- SuppFig1.jpg 